\author{
CONVEX AND FRACTAL GEOMETRY \\ BANACH CENTER PUBLICATIONS, VOLUME 84 \\ INSTITUTE OF MATHEMATICS \\ POLISH ACADEMY OF SCIENCES \\ WARSZAWA 2009
}

\title{
WALK DIMENSION AND FUNCTION SPACES ON SELF-SIMILAR FRACTALS
}

\author{
KATARZYNA PIETRUSKA-PAŁUBA \\ Institute of Mathematics, University of Warsaw \\ Banacha 2, 02-097 Warszawa, Poland \\ E-mail:kpp@mimuw.edu.pl
}

\begin{abstract}
We outline the construction of Brownian motion on certain self-similar fractals and introduce the notion of walk dimension. We then show how the probabilistic approach relates to the theory of function spaces on fractals.
\end{abstract}

1. Introduction. In the late 80 's, there emerged a need to construct the Brownian motion on fractal structures. By 'Brownian motion' we understand here a diffusion which is invariant under the action of local isometries of the state-space. This construction has been successfully performed on a class of highly regular self-similar fractals, called the nested fractals (their definition is due to Lindstrøm [22]). Over the years, several approaches have been worked out, and finally in 1996 Sabot [29] proved that the Brownian motion on nested fractals is unique up to a linear time-change.

On non-nested fractals such as the Sierpiński carpet, the results are far less satisfactory. Although the Brownian motion has been constructed by Barlow and Bass [2], and its nice properties have been established, it is only defined as a limit point of certain sequence, through a tightness argument. Its uniqueness is still a conjecture.

In connection with the Brownian motion, one introduces a qualitative characteristic of the fractal - the so-called walk dimension. It manifests as the space/time scaling of the Brownian motion, but also can be defined on purely analytic grounds, without appealing to any stochastic process. The walk dimension appears as a threshold in certain Besovtype function spaces. The two coincide when the set in question supports a nice diffusion (this is the case of nested fractals, the Sierpiński carpet, and many other spaces).

This survey paper is organized as follows. First, we define the nested-fractals (Section 2,3) and we discuss the construction of Brownian motion there, together with its

2000 Mathematics Subject Classification: 60J35, 46E30.

Key words and phrases: fractals, fractional diffusion, walk dimension.

Research partially supported by KBN grant 1-PO3A-008-29.

The paper is in final form and no version of it will be published elsewhere. 
properties (Section 4,5). Here the walk dimension is introduced probabilistically. Finally, we turn our attention to the general setting. We determine the consequences pertaining to the function spaces (and to the walk dimension in particular) that can be drawn from the existence of a nice process. See Section 6,7 . We do not present proofs, but always indicate where they can be found.

2. Iterated function systems and the dimension of the attractor. The material of this section follows $[5,8,15]$.

Let $(X, \delta)$ be a given metric space.

Definition 1. Suppose $\phi_{i}: X \rightarrow X, i=1,2, \ldots, M$ are contractions, with respective contraction factors $0<s_{i}<1$. The system $\left\{\phi_{1}, \phi_{2}, \ldots, \phi_{M}\right\}$ is called a (hyperbolic) iterated function system (IFS) on $(X, \delta)$.

For any nonempty compact set $B \subset X$ let

$$
\Phi(B)=\phi_{1}(B) \cup \cdots \cup \phi_{M}(B) \in \mathcal{H}(X) .
$$

Then $\Phi$ is a contraction of the space $\mathcal{H}(X)$ of all nonempty compact subsets of $X$, equipped with the Hausdorff distance, with contraction factor $s=\max _{i} s_{i}$. By a simple application of the Banach fixed point theorem we obtain

Theorem 1 ([15]). There exists a unique set $A \in \mathcal{H}(X)$ such that $\Phi(A)=A$. Moreover, for any $B \in \mathcal{H}(X)$ one has $\lim _{n \rightarrow \infty} \Phi^{n}(B)=A$, the limit being taken in the Hausdorff distance.

The set $A$ is called the attractor of the IFS $\left\{\phi_{1}, \ldots, \phi_{M}\right\}$.

Example 1. If $X=\mathbf{R}, \phi_{1}(x)=\frac{1}{2} x, \phi_{2}(x)=\frac{1}{2} x+\frac{1}{2}$, then $A=[0,1]$.

ExAmple 2. If $X=\mathbf{R}^{2}, \phi_{1}(x)=\frac{1}{2} x, \phi_{2}(x)=\frac{1}{2} x+\left(\frac{1}{2}, 0\right), \phi_{3}(x)=\frac{1}{2} x+\left(\frac{1}{4}, \frac{\sqrt{3}}{4}\right)$, then $A$ is the Sierpiński gasket (triangle).

ExAmple 3. If $X=\mathbf{R}^{2}, M=8, \phi_{i}(x)=\frac{1}{3} x+a_{i}$, where $a_{1}=(0,0), a_{2}=\left(\frac{1}{3}, 0\right)$, $a_{3}=\left(\frac{2}{3}, 0\right), a_{4}=\left(0, \frac{1}{3}\right), a_{5}=\left(\frac{2}{3}, \frac{1}{3}\right), a_{6}=\left(0, \frac{2}{3}\right), a_{7}=\left(\frac{1}{3}, \frac{2}{3}\right), a_{8}=\left(\frac{2}{3}, \frac{2}{3}\right)$, then $A$ is the Sierpiński carpet.

It is usually hard to compute, or even estimate, the Hausdorff dimension of the attractor, $d_{H}(A)$. Instead we can try to investigate its box-counting (Minkowski, fractal) dimension:

$$
d_{F}(A)=\limsup _{\varepsilon \rightarrow 0} \frac{\log \mathcal{N}(A, \varepsilon)}{\log \frac{1}{\varepsilon}},
$$

where for $\varepsilon>0, \mathcal{N}(A, \varepsilon)$ is the smallest possible number of closed balls with radius $\varepsilon$ needed to cover $A$. It is more manageable than the Hausdorff dimension and ready for computer application, but still difficult to work with.

Note that in the literature concerning Brownian motion on fractals (see e.g. [1, 4]) it is customary to call this dimension 'the fractal dimension', and to denote it by $d_{f}$.

The 'size' of the attractor of an IFS can also be measured using the so-called similarity dimension, $D(A)$ : it is the unique solution of the equation

$$
l(d):=s_{1}^{d}+\cdots+s_{M}^{d}=1 .
$$


Since $l$ is continuous and monotone decreasing, $l(0)=M$, and $l(\infty)=0$, such a solution exists and is unique. In general, one compact subset of $X$ can have several representations as the attractor of an IFS, and different IFS's can yield distinct similarity dimensions. For example, if one adds the mapping $\phi_{3}(x)=\frac{1}{2} x+\frac{1}{4}$ to the system from Example 1 , one still gets $[0,1]$ as the attractor, but now $D(A)=\frac{\log 3}{\log 2}$.

To rectify this situation, the open set condition is introduced. This condition ensures that there are no essential overlaps between parts of the attractor.

Definition 2 (Open Set Condition). Let $\left\{\phi_{1}, \ldots, \phi_{M}\right\}$ be an IFS. We say that the open set condition holds for this IFS if there exists an open, nonempty set $V \subset X$ such that for $i \neq j$ we have $\phi_{i}(V) \cap \phi_{j}(V)=\emptyset$, and

$$
\Phi(V)=\bigcup_{i=1}^{M} \phi_{i}(V) \subset V .
$$

Then we have the following.

THEOREM 2. Let $A$ be the attractor of an iterated functions system $\left\{\phi_{1}, \ldots, \phi_{M}\right\}$. Then:

1. $d_{H}(A) \leq d_{F}(A) \leq D(A)$.

2. When the IFS satisfies the open set condition, then all three dimensions are equal.

3. Nested fractals. In this section, we will have $X=\mathbf{R}^{N}$, and $\delta$ - the Euclidean distance. Suppose $\phi_{1}, \phi_{2}, \ldots, \phi_{M}: \mathbf{R}^{N} \rightarrow \mathbf{R}^{N}$ are similitudes with common scaling factor $L>1$, i.e. $\phi_{i}(x)=(1 / L) U_{i}(x)+v_{i}$, where $U_{i}$ are isometries of $\mathbf{R}^{N}$ and $v_{i} \in \mathbf{R}^{N}$ are fixed translation vectors.

Definition 3. The attractor of the system $\left\{\phi_{1}, \ldots, \phi_{M}\right\}$, i.e. the unique nonempty compact set $\mathcal{K}$ such that

$$
\Phi(\mathcal{K})=\bigcup_{i=1}^{M} \phi_{i}(\mathcal{K})=\mathcal{K}
$$

is called the self-similar fractal generated by the family $\left\{\phi_{1}, \ldots, \phi_{M}\right\}$.

$\mathcal{K}$ is compact, and in particular has finite diameter.

To proceed with the definition of a nested fractal, we have to analyse the set of fixed points of the similitudes $\phi_{i}$. Let $F$ be the collection of fixed points of the $\phi_{i}^{\prime} s(\# F \leq M)$. Then $x \in F$ is called an essential fixed point if there exists another fixed point $y \in F$ and two different transformations $\phi_{i}, \phi_{j}$ such that $\phi_{i}(x)=\phi_{j}(y)$. The collection of all essential fixed points is denoted by $V^{\langle 0\rangle}$.

'Small' parts of the set $\mathcal{K}$ that are similar to $\mathcal{K}$ are called complexes:

Definition 4. Take $m \in \mathbf{Z}_{+}$. Any set of the form $S=\phi_{i_{1}} \circ \cdots \circ \phi_{i_{m}}(\mathcal{K}), i_{1}, \ldots, i_{m} \in$ $\{1, \ldots, M\}$, is called an $m$-complex. For any such $S$, its vertices are the points from the set $V(S)=\phi_{i_{1}} \circ \cdots \circ \phi_{i_{m}}\left(V^{\langle 0\rangle}\right)$.

All vertices of all $m$-complexes constitute the $m$-grid $V^{\langle m\rangle} \subset \mathcal{K}$. Further, let $V^{\langle\infty\rangle}=\bigcup_{m=0}^{\infty} V^{\langle m\rangle}$ and $\mathcal{K}=\overline{\bigcup_{m=0}^{\infty} V^{\langle m\rangle}}$. 
The following definition is due to Lindstrøm [22]:

Definition 5. The attractor $\mathcal{K}$ of an IFS $\left\{\phi_{1}, \ldots, \phi_{M}\right\}$ is called a nested fractal if $\# V^{\langle 0\rangle} \geq 2$, the open set condition is satisfied, and the following three conditions hold true.

(C1) (Symmetry) For $x, y \in V^{\langle 0\rangle}$ let $R_{x, y}$ be the reflection in the hyperplane bisecting the segment $[x, y]$. We stipulate that

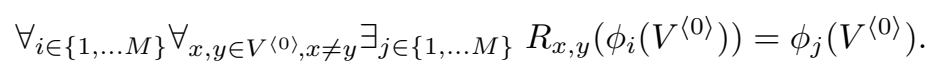

(C2) (Connectivity) On the set $V_{1}^{\langle 0\rangle} \stackrel{\text { def }}{=} \bigcup_{i=1}^{M} \phi_{i}\left(V^{\langle 0\rangle}\right)$ define the graph structure $E_{(1)}$ as follows: we say that $(x, y) \in E_{(1)}$ if and only if $x$ and $y$ belong to the same 1-complex. Then we require the graph $\left(V^{\langle 1\rangle}, E_{(1)}\right)$ to be connected.

(C3) (Nesting) Suppose $S, T$ are two different 1-complexes. Then $S \cap T=V(S) \cap V(T)$.

The Sierpiński gasket is a nested fractal, the Sierpiński carpet is not: on the carpet, the nesting property is violated.

Because of Theorem 2, the Hausdorff dimension of the set $\mathcal{K}$ is equal to

$$
d_{F}=d_{F}(\mathcal{K})=\frac{\log M}{\log L} .
$$

The Hausdorff measure in dimension $d_{F}$, denoted by $\mu$, serves as the natural measure on $\mathcal{K}$. For later use, observe that $\mathcal{K}$ is a $d_{F}$-set (or, equivalently, that the measure $\mu$ is Ahlfors $d_{F}$-regular), i.e. for any ball with respect to the Euclidean metric,

$$
A_{1} r^{d_{F}} \leq \mu(B(x, r)) \leq A_{2} r^{d_{F}}
$$

where $A_{1}, A_{2}$ are two positive constants independent of $x, r$.

4. Random walks and Brownian motion on fractals. Fractal-like structures are abundant in physical applications and from the physicists' point of view it is easy to construct a 'Brownian motion' on these sets. It should be the 'natural' random movement with no jumps that a particle would perform if no external force is present. Such a random movement strongly depends on the geometry of this set: for example, the more 'holes' (= obstacles) in the medium, the more difficult it should be to move - and consequently the movement should be slowed down. For the physical motivation, the reader should consult e.g. [28]. It turns out that the Hausdorff dimension alone is not sensitive enough to determine how the process moves.

The rigorous mathematical construction of the Brownian motion on fractal sets has been restricted so far to the class of nested fractals and their generalizations called postcritically finite self-similar sets (introduced by Kigami [18]; these sets are not necessarily embedded in $\mathbf{R}^{N}$ ). The only work beyond this class pertains to the Brownian motion on the carpet [2]: the 'Brownian motion' has been constructed and its properties have been analysed. Its uniqueness remains an open problem.

Early constructions on the Sierpiński gasket (due to Goldstein [12], Kusuoka [21] and Barlow-Perkins [4]) used its discrete approximations by $m$-grids. The gasket $\mathcal{T}$ these papers dealt with was not understood as the attractor of a three-map IFS of Example 2, 
but as the closure of the infinitely extended $m$-grids, which we shall denote by $\mathcal{T}^{\langle m\rangle}$. Every point in $\mathcal{T}^{\langle m\rangle}$ has four natural neighbors, at distance $\frac{1}{2^{m}}$ apart. On this set, one defines the nearest-neighbor random walk (NNRW) denoted by $\left(X_{n}^{\langle m\rangle}\right)_{n \geq 0}$ : every minute, the process jumps with equal probability to one of the four neighboring sites. As the grids get finer and finer, the expected time needed to move at distance 1 increases to infinity. In order to pass to the limit, one needs to speed up these random walks, so that the expected time needed to traverse the unit triangle would remain equal to 1 . After appropriate scaling (which is tantamount to the time-scaling factor being equal to 5), one gets:

$$
\left(X_{\left[5^{m} t\right]}^{\langle m\rangle}, t \geq 0\right) \Rightarrow\left(X_{t}, t \geq 0\right)
$$

almost surely and uniformly in $t$. This limiting proces exhibits a large amount of symmetry and can be shown to be unique among all diffusions on the gasket that are invariant under the group of local symmetries of the gasket. It is therefore called the Brownian motion on the Sierpiński gasket.

This definition (with no uniqueness proven at that time) was later generalized to nested fractals by T. Lindstrøm [22]. Although the limit procedure was similar to that of [4], the main tool there was the nonstandard analysis.

4.1. The walk dimension. The scaling for the random walk on the unbounded Sierpiński gasket as in [4], needed to obtain the desired convergence, was the multiplication by 5 every time we went into the next finer grid. It means that the NNRW on the gasket needs in average 5 units of time to travel twice the unit distance. More precisely, if we start the random walk $X_{n}^{\langle 1\rangle}$ from 0 and run until it hits other vertices of one of the unit triangles meeting at 0 , then the expected lifetime of this process equals to 5 . Due to nesting, this property carries over to subsequent approximations, and further to the limiting continuous process: when started from zero, $2 X_{t}$ and $X_{5 t}$ have the same law.

The quantity $d_{w}=d_{w}(\mathcal{T})=\frac{\log 5}{\log 2}$ is called the walk dimension of the Sierpinski gasket. It governs the time/space scaling of the Brownian motion on the gasket. The number $d_{w}$ enters the transition density estimates for the process $\left(X_{t}\right)$ in the following way.

THEOREM $3([4])$. The process $X_{t}$ (on the unbounded Sierpinski gasket $\mathcal{T}$ ) is a strong Markov, Feller process, and has a symmetric transition density $p(t, x, y)$, with respect to the Hausdorff measure $\mu$. This transition density satisfies the following estimate:

$$
\frac{c_{1}}{t^{d_{F} / d_{w}}} \exp \left\{-c_{2}\left(\frac{|x-y|}{t^{1 / d_{w}}}\right)^{\frac{d_{w}}{d_{w-1}}}\right\} \leq p(t, x, y) \leq \frac{c_{3}}{t^{d_{F} / d_{w}}} \exp \left\{-c_{4}\left(\frac{|x-y|}{t^{1 / d_{w}}}\right)^{\frac{d_{w}}{d_{w}-1}}\right\}
$$

where $|\cdot-\cdot|$ denotes the Euclidean distance.

For the Sierpiński gasket in $\mathbf{R}^{N}$, its Hausdorff dimension is equal to $\frac{\log (N+1)}{\log 2}$, and its walk dimension to $\frac{\log (N+3)}{\log 2}$.

Although this hands-on construction permitted also to define the Brownian motion on nested fractals (e.g. Lindstrøm [22]), the Dirichlet forms approach which we discuss in the next section is much simpler. 
5. Dirichlet forms and the Brownian motion. Another approach, particularly wellfitted to accommodate the self-similarity and the symmetries of nested fractals, is the Dirichlet form construction of the Brownian motion. It also proved useful in establishing uniqueness of the desired process.

Suppose $\mathcal{K}$ is a nested fractal being the attractor of the IFS $\left\{\phi_{1}, \ldots, \phi_{M}\right\}$ in $\mathbf{R}^{N}$, with scaling factor $L$. The goal is to construct a symmetric, bilinear, nonnegative form $\mathcal{E}(f, f)$ on $L^{2}(\mathcal{K}, \mu)$, which is densely defined, closed, Markovian, regular, and agrees with the symmetries of $\mathcal{K}$. Then the process will be defined as well (see [10]).

In the fractal setting, this construction was first performed by Fukushima ans Shima [11] on the Sierpiński gasket. Even in this simplest case, the important features of the construction were present. The construction was extended to general nested fractals by Fukushima [9]. Our presentation follows that of Barlow [1], where the case of p.c.f. selfsimilar sets is covered as well.

Recall that by $V^{\langle 0\rangle}$ we denoted the collection of all the essential fixed points of the IFS associated with $\mathcal{K}$. Let $A=\left(a_{x, y}\right)_{x, y \in V\langle 0\rangle}$ be a real-valued matrix such that:

1. for all $x, y \in V^{\langle 0\rangle}, x \neq y, a_{x, y} \geq 0$ and $a_{x, y}=a_{y, x}$;

2. for all $x \in V^{\langle 0\rangle}, \sum_{y} a_{x, y}=0$

(such a matrix is called a conductivity matrix), and define

$$
C\left(V^{\langle 0\rangle}\right) \ni f \mapsto \mathcal{E}_{A}^{\langle 0\rangle}(f, f)=\frac{1}{2} \sum_{x, y \in V^{\langle 0\rangle}} a_{x, y}(f(x)-f(y))^{2} .
$$

Next, introduce two operators acting on Dirichlet forms:

reproduction, $\mathcal{R} e$ : for a Dirichlet form $\mathcal{E}^{\langle 0\rangle}$ on $C\left(V^{\langle 0\rangle}\right)$, let $\mathcal{R} e(\mathcal{E})(f, f)=\widetilde{\mathcal{E}}^{\langle 1\rangle}(f, f)=$ $\sum_{i=1}^{M} \mathcal{E}^{\langle 0\rangle}\left(f \circ \phi_{i}, f \circ \phi_{i}\right)$ be a bilinear form on $C\left(V^{\langle 1\rangle}\right)$.

decimation, $\mathcal{D} e$ : for a given bilinear form on $C\left(V^{\langle 1\rangle}\right)$,

$$
\mathcal{D} e(\mathcal{E})(f, f)=\left.\mathcal{E}\right|_{V^{\langle 0\rangle}}(f, f)=\inf \left\{\mathcal{E}(g, g): g: V^{\langle 1\rangle} \rightarrow \mathbf{R} \text { and }\left.g\right|_{V^{\langle 0\rangle}}=f\right\} .
$$

The key property linking these two operators is the following:

TheOREM 4. Suppose $\mathcal{K}$ is a nested fractal. There exists a unique number $\rho=\rho(\mathcal{K})>1$ and a unique (up to a multiplicative constant) irreducible conductivity matrix $A$ on $V^{\langle 0\rangle}$, such that $\mathcal{D} e \circ \mathcal{R} e\left(\mathcal{E}_{A}\right)=\frac{1}{\rho} \mathcal{E}_{A}$.

This result is due to Lindstrøm [22] in its existence part, and to Sabot [29] in its uniqueness part. This uniqueness implies the uniqueness of the Brownian motion on $\mathcal{K}$.

Choose this matrix $A$ and define $\mathcal{E}^{\langle 0\rangle}=\mathcal{E}_{A}^{\langle 0\rangle}$, then let

$$
\widetilde{\mathcal{E}}^{\langle m\rangle}(f, f)=\sum_{i_{1}, \ldots, i_{m}} \mathcal{E}^{\langle 0\rangle}\left(f \circ \phi_{i_{1}} \circ \cdots \circ \phi_{i_{m}}, f \circ \phi_{i_{1}} \circ \cdots \circ \phi_{i_{m}}\right),
$$

and further

$$
\mathcal{E}^{\langle 1\rangle}(f, f)=\rho \widetilde{\mathcal{E}}^{\langle 1\rangle}(f, f) ; \quad \mathcal{E}^{\langle m\rangle}(f, f)=\rho^{m} \widetilde{\mathcal{E}}^{\langle m\rangle}(f, f) .
$$

Multiplication by $\rho$ in every step makes the sequence $\mathcal{E}^{\langle m\rangle}$ nondecreasing, and so we obtain the following: 
TheOREM 5 ([1, 9]). For every $f: V^{\langle\infty\rangle} \rightarrow \mathbf{R}$, and every $m=0,1,2, \ldots$ one has

$$
\mathcal{E}^{\langle m\rangle}(f, f) \leq \mathcal{E}^{\langle m+1\rangle}(f, f) .
$$

Set $\widetilde{\mathcal{D}}=\left\{f: V^{\langle\infty\rangle} \rightarrow \mathbf{R}: \sup _{m} \mathcal{E}^{\langle m\rangle}(f, f)<\infty\right\}$ and for $f \in \widetilde{\mathcal{D}}$

$$
\widetilde{\mathcal{E}}(f, f)=\lim _{m \rightarrow \infty} \mathcal{E}^{\langle m\rangle}(f, f),
$$

and further $\mathcal{D}=\left\{f \in C(\mathcal{K}):\left.f\right|_{V\langle\infty\rangle} \in \widetilde{\mathcal{D}}\right\}, \mathcal{E}(f, f)=\widetilde{\mathcal{E}}\left(\left.f\right|_{V^{\langle\infty\rangle}},\left.f\right|_{V^{\langle\infty\rangle}}\right)$ for $f \in \mathcal{D}$. $(\mathcal{E}, \mathcal{D})$ is a regular Dirichlet form on $L^{2}(\mathcal{K}, \mu)$, which agrees with the group of local symmetries of $\mathcal{K}$.

5.1. Significance of the constant $\rho$. By analogy with electrical circuit theory, $\rho$ is called the resistance scaling factor. Define the walk dimension:

$$
d_{w}=d_{w}(\mathcal{K})=\frac{\log M \rho}{\log L}>1 .
$$

It agrees with the formerly introduced definition. Also, one has

$$
\rho=\frac{L^{d_{w}}}{L^{d_{F}}}
$$

Next,

$$
d_{s}=d_{s}(\mathcal{K})=\frac{2 d_{F}}{d_{w}}
$$

is called the spectral dimension of the set $\mathcal{K}$. As long as $\rho>1$, we would have $d_{s}<2$.

\section{Transition density, the domain of the Dirichlet form, and the walk di-} mension revisited. The Dirichlet form constructed by means of the above Theorem corresponds to a strong Markov, Feller process $\left(X_{t}\right)$ which has a transition density with respect to the Hausdorff measure on $\mathcal{K}$ (in dimension $d_{F}=\frac{\log M}{\log L}$ ). Although this process was defined differently, its transition density satisfies (2), with appropriate values of $d_{F}, d_{w}$.

The Brownian motion on the Sierpiński carpet, as constructed in [2], also shares this property: its transition density fulfills (2).

It turns out that this form of estimate is typical for a diffusion on general metric spaces, not necessarily embedded in $\mathbf{R}^{N}$. Suppose $(X, \delta, \mu)$ is a metric measure space, i.e. a locally compact space with metric $\delta$, equipped with an Ahlfors $d_{F}$-regular measure, $d_{F} \geq 1$. Suppose that $X$ satisfies the chain condition: for any two points $x, y \in X$ and any number $n \in \mathbf{Z}_{+}$, there exists a constant $C>0$ and a chain $x=x_{0}, x_{1}, \ldots, x_{n}=y$ s.t. $\delta\left(x_{i}, x_{i+1}\right) \leq \frac{C}{n} \delta(x, y)$, with $C$ independent of $x, y$.

Then we have the following definition (Barlow [1]):

Definition 6. A Markov process $\left(X_{t}\right)$ on $(X, \delta, \mu)$ is called a fractional diffusion on $X$ if it is a conservative $\mu$-symmetric Feller diffusion, and it has a transition density $p(t, x, y)$, jointly continuous in $(x, y)$ for any given $t>0$, which satisfies

$$
\frac{c_{1}}{t^{\alpha}} \exp \left\{-\frac{c_{2} \delta(x, y)^{\beta \gamma}}{t^{\gamma}}\right\} \leq p(t, x, y) \leq \frac{c_{3}}{t^{\alpha}} \exp \left\{-\frac{c_{4} \delta(x, y)^{\beta \gamma}}{t^{\gamma}}\right\} \text {. }
$$


The estimate (4) is only apparently more general that (2). If $d_{H}(X)=d_{F}$, then necessarily $\alpha=\frac{d_{F}}{\beta}, \beta>1, \gamma=\frac{1}{\beta-1}$.

We have already seen that the Brownian motion on the Sierpinski gasket is a fractional diffusion. Similar estimates have been proven for nested fractals and p.c.f.s.s. sets (Kumagai [19], Hambly-Kumagai [17]). Other examples are furnished by the Brownian motion on manifolds, see [31].

A natural question arising in this approach was to identify the domain of the Dirichlet form of such a process. It has been done first on the Sierpiński gasket [16], then on nested fractals [24], and on general metric spaces [25, 20, 13]. It was noticed in [25] that the only relevant feature here is the transition density estimate: having this, we can prove that the domain of the Dirichlet form is the Besov-Lipschitz space $\operatorname{Lip}\left(d_{w} / 2,2, \infty\right)(X)$.

This space is defined as follows. For $f \in L^{2}(X, \mu)$, let

$$
a_{n}(f)=2^{n\left(d_{F}+d_{w}\right)} \iint_{\delta(x, y) \leq 2^{-n}}|f(x)-f(y)|^{2} \mathrm{~d} \mu(x) \mathrm{d} \mu(y) .
$$

Then $f \in \operatorname{Lip}\left(d_{w} / 2,2, \infty\right)(X)$ if and only if $\sup _{n} a_{n}(f)<\infty$. This space is a Banach space when equipped with the norm

$$
\|f\|_{L i p}=\|f\|_{2}+\left(\sup _{n} a_{n}(f)\right)^{1 / 2} .
$$

We have the following.

Theorem 6. ([16, 25]). Let $(X, \delta, \mu)$ be a measure metric space, where $\mu$ is a $d_{F}$-measure. Suppose that $X$ is the state space of a reversible Markov process with transition density satisfying (2). Then $\mathcal{D}(\mathcal{E})=\operatorname{Lip}\left(d_{w} / 2,2, \infty\right)(X)$. Moreover, for $f \in \mathcal{D}(\mathcal{E})$ we have $\frac{1}{C} \mathcal{E}(f, f) \leq \sup _{n} a_{n}(f) \leq C \mathcal{E}(f, f)$, with certain constant $C$ not depending on $f$.

Efforts have been made to obtain similar results for diffusions with transition density obeying a more general estimate: where the exponential function in (2) would be replaced with a more general, decreasing function $\Phi$. It turned out that such a situation is not possible: if the estimate is of the form $c t^{-d_{F} / d_{w}} \Phi\left(\frac{\delta(x, y)}{t^{1 / d_{w}}}\right)$, and $X$ satisfies the chain condition, then the process is a diffusion if and only if $\Phi$ is of exponential type. See a recent paper of Grigor'yan and Kumagai [14].

6.1. The walk dimension revisited. In addition to the characterization of the domain of the Dirichlet form, it has been proven in $[16,24,25]$ that once the space $(X, \delta, \mu)$ supports a diffusion with transition density satisfying (2), then the spaces $\operatorname{Lip}(\gamma, 2, \infty)(X)$ became all trivial for $\gamma>d_{w} / 2$. On the other hand, since $\operatorname{Lip}\left(d_{w} / 2,2, \infty\right)(X)$ is the domain of a Dirichlet form, it is a dense subspace of $L^{2}(X, \mu)$. In particular it means that on a given space $X$, all diffusions satisfying (2) must share the exponent $d_{w}$. We therefore call $d_{w}$ the walk dimension of $X$. The argument above also proves that

$$
d_{w}(X)=\sup \left\{\gamma>0: \operatorname{Lip}(\gamma / 2,2, \infty)(X) \text { is a dense subspace of } L^{2}(X, \mu)\right\} .
$$

We have previously seen that for the Sierpiński gasket in $\mathbf{R}^{N}$, the walk dimension equals to $\frac{\log (N+3)}{\log 2}$, and for a general nested fractal, $d_{w}=\frac{\log (M \rho)}{\log L}$, where $\rho$ is the constant from Theorem 4. Also, the walk dimension of $\mathbf{R}^{N}$ is equal to 2 (for the usual Brownian motion, $d_{w}=2$ ). In general it is known (see $\left.[1,13]\right)$ that $2 \leq d_{w} \leq d_{F}+1$. 
The Sierpiński carpet is not a nested fractal, and the two approaches presented above do not work. Nevertheless, a diffusion satisfying (2) has been constructed ([2]), and so it is plausible to ask what is the value of the walk dimension of the carpet.

Problem 1. Determine the value of the walk dimension of the Sierpinski carpet. For the two-dimensional carpet, [3] gives a numerical approximation $d_{w} \approx \frac{\log 10.012}{\log 3}$, but the exact value is unknown.

7. Function spaces on fractals. So far, the walk dimension has been introduced in the following three ways:

(1) as the time/space scaling of the nearest neighbor random walk on approximations of nested fractals;

(2) through the resistance scaling factor;

(3) as the threshold for the Besov-Lipschitz spaces $\operatorname{Lip}(\gamma, 2, \infty)(X)$.

Another 'threshold' property is present in the family of Besov-Slobodeckii spaces on $(X, \delta, \mu)((X, \delta, \mu)$ is as in the previous section):

$$
\Lambda^{2, \alpha}(X)=\left\{f \in L^{2}(, \mu): \int_{X} \int_{X} \frac{|f(x)-f(y)|^{2}}{\delta(x, y)^{d_{F}+\alpha}} \mathrm{d} \mu(x) \mathrm{d} \mu(y):=E^{(\alpha)}(f, f)<\infty\right\} .
$$

It has been proven in [26] that when $\alpha \geq d_{w}$, then these spaces are trivial. When $\alpha<d_{w}$, and $X$ is e.g. a compact nested fractal, then the spaces $\Lambda^{2, \alpha}(X)$ are dense in $L^{2}(X, \mu)$ : this is so because $\operatorname{Lip}(\beta, 2, \infty)(X) \subset \Lambda^{2, \alpha}(X)$ when $\alpha<2 \beta$.

Namely, we have $([26])$

THEOREM 7. Suppose that $f \in \Lambda^{2, d_{w}}(X)$, where $X$ is a $d_{F}$-set carrying a diffusion for which the estimate (3) holds. Then $f \equiv$ const a.e.

Therefore the walk dimension has yet another characterization:

$$
d_{w}=\sup \left\{\alpha>0: \Lambda^{2, \alpha}(X) \text { is a dense subspace of } L^{2}(X, \mu)\right\} .
$$

Recall that $d_{w} \geq 2$, and that in the examples already discussed we had $d_{w}>2$. Therefore there are nonconstant functions in the Besov-Lipschitz as well as Besov-Slobodeckii spaces in the range $1<\gamma \leq d_{w} / 2$ (resp. $2 \leq \alpha<d_{w}$ ). This holds true even for $X \subset \mathbf{R}^{N}$, which comes as a bit of a surprise - since $d_{w}\left(\mathbf{R}^{N}\right)=2$ is the threshold value for $\mathbf{R}^{N}$, we see that it is possible that the threshold for a subset is actually placed higher than that of the complete space! We know that when $\alpha<2$, then the Besov-Slobodeckii spaces on $X$ are traces of appropriate Besov spaces on $\mathbf{R}^{N}$. What happens for $2 \leq \alpha<d_{w}$ is unknown.

Problem 2. Analyse the spaces $\Lambda^{2, \alpha}(X)$ for $X \subset \mathbf{R}^{N}$ when $2 \leq \alpha<d_{w}$.

When the space $(X, \delta, \mu)$ supports a diffusion, it is known that $E^{(\alpha)}$ are regular Dirichlet forms up to $\alpha=d_{w}$ (excluding $\left.\alpha=d_{w}\right)$.

Problem 3. Prove that $E^{(\alpha)}$ are regular Dirichlet forms for $\alpha<d_{w}$, without assuming the existence of a diffusion satisfying (2).

This has been done up to $\alpha=2([30,7])$. 
When $X=\Omega$ is a nice domain in $\mathbf{R}^{N}$, then the Besov-Lipschitz spaces are limits of Besov-Slobodeckii spaces in the following sense (see $[6,23])$ :

$$
\lim _{\alpha \nearrow^{2}}(2-\alpha) \int_{\Omega} \int_{\Omega} \frac{|f(x)-f(y)|^{p}}{|x-y|^{N+\alpha \frac{p}{2}}} \mathrm{~d} x \mathrm{~d} y=c_{p} \cdot \int_{\Omega}|\nabla f|^{p} \mathrm{~d} x .
$$

For $p=2, \int_{\Omega}|\nabla f|^{2} \mathrm{~d} x$ is the Dirichlet form for the Brownian motion on $\Omega$.

In general metric spaces, the expressions (5) are not expected to converge when $\alpha$ approaches the critical value $d_{w}$. However, we have been able to establish the following:

THEOREM $8([27])$. Suppose that $(X, \delta, \mu)$ is a measure metric space supporting a diffusion satisfying (2), and let $\mathcal{E}(f, f)$ be the Dirichlet form associated with that diffusion. Then for any $f \in \mathcal{D}(\mathcal{E})\left(=\operatorname{Lip}\left(d_{w} / 2,2, \infty\right)(X)\right)$ we have:

$$
C_{1} \mathcal{E}(f, f) \leq \liminf _{\alpha \nearrow d_{w}}\left(d_{w}-\alpha\right) E^{(\alpha)}(f, f) \leq \limsup _{\alpha \nearrow d_{w}}\left(d_{w}-\alpha\right) E^{(\alpha)}(f, f) \leq C_{2} \mathcal{E}(f, f) .
$$

The proof uses probabilistic features relative to stable processes on $d$-sets. How to do this without appealing to stochastic processes is unknown so far.

\section{References}

[1] M. T. Barlow, Diffusion on fractals, in: Lectures on Probability and Statistics, Ecole d'Eté de Prob. de St. Flour XXV-1995, Lecture Notes in Mathematics 1690, Springer-Verlag, New York, 1998, 1-121.

[2] M. T. Barlow and R. F. Bass, Brownian motion and analysis on Sierpiński carpets, Canadian J. Math. 51 (1999), 673-744.

[3] M. T. Barlow, R. F. Bass and J. D. Sherwood, Resistance and spectral dimension on Sierpinski carpets, J. Phys. A 23 (1990), L253-L258.

[4] M. T. Barlow and E. A. Perkins, Brownian motion on the Sierpinski gasket, Prob. Th. Rel. Fields 79 (1988), 543-623.

[5] M. Barnsley, Fractals Everywhere, Academic Press, 1988.

[6] J. Bourgain, H. Brézis and P. Mironescu, Another look at Sobolev spaces, in: Optimal Control and PDE. In honour of Prof. A. Bensoussan 60th Birthday, J. L. Menaldi et al. (eds.), IOS Press, Amsterdam, 2001.

[7] Z. Q. Chen and T. Kumagai, Heat kernel estimates for jump processes of mixed types on metric measure spaces, Prob. Th. Rel. Fields 140 (2008), 277-317.

[8] K. Falconer, Geometry of Fractal Sets, Cambridge Univ. Press, 1985.

[9] M. Fukushima, Dirichlet forms, diffusion processes and spectral dimensions for nested fractals, in: Ideas and Methods in Stochastic Analysis, Stochastics and Applications, Cambridge Univ. Press, Cambridge, 1992, 151-161.

[10] M. Fukushima, Y. Oshima and M. Takeda, Dirichlet Forms and Symmetric Markov Processes, de Gruyter, Berlin, 1994.

[11] M. Fukushima and T. Shima, On a spectral analysis for the Sierpinski gasket, J. Pot. Anal. 1 (1992), 1-35.

[12] S. Goldstein, Random walk and diffusion on fractals, in: H. Kesten (ed.), Percolation Theory and Ergodic Theory of Infinite Particle Systems, IMA Math. Appl. 8, Springer, New York, 1987, 121-129. 
[13] A. Grigoryan, J. Hu and K. S. Lau, Heat kernels on metric measure spaces and an application to semilinear elliptic equations, Trans. Amer. Math. Soc. 355 (2003), 2065-2095.

[14] A. Grigoryan and T. Kumagai, On the dichotomy in the heat kernel two sided-estimates, in: Analysis on Graphs and its Applications, P. Exner et al. (eds.), Proc. Sympos. Pure Math. 77, Amer. Math. Soc., 2008, 199-210.

[15] J. E. Hutchinson, Fractals and self-similarity, Indiana Univ. Math. J. 30 (1981), 713-747.

[16] A. Jonsson, Brownian motion on fractals and function spaces, Math. Z. 222 (1996), 495504.

[17] B. M. Hambly and T. Kumagai, Transition density estimates for diffusion processes on post critically finite self-similar fractals, Proc. London Math. Soc. 78 (1999), 431-458.

[18] J. Kigami, A harmonic calculus on p.c.f. self-similar sets, Trans. AMS 335 (1993), 721755 .

[19] T. Kumagai, Estimates of the transition densities for Brownian motion on nested fractals, Prob. Th. Rel. Fields 96 (1993), 205-224.

[20] T. Kumagai, Brownian motion penetrating fractals - An application of the trace theorem of Besov spaces, J. Funct. Anal. 170 (2000), 69-92.

[21] S. Kusuoka, A diffusion process on a fractal, in: K. Itô and N. Ikeda (eds.), Symposium on Probabilistic Methods in Mathematical Physics, Academic Press, Amsterdam, 1987, 251-274.

[22] T. Lindstrøm, Brownian motion on nested fractals, Mem. AMS 420 (1990).

[23] W. Mazja and J. Nagel, Über äquivalente Normierung der anisotropen Funktionalräume $H^{\mu}\left(\mathbf{R}^{n}\right)$, Beiträge zur Analysis 12 (1978), 7-17.

[24] K. Pietruska-Pałuba, Some function spaces related to the Brownian motion on simple nested fractals, Stoch. Stoch. Rep. 67 (1999), 267-285.

[25] K. Pietruska-Pałuba, On function spaces related to fractional diffusions on d-sets, Stoch. Stoch. Rep. 70 (2000), 153-164.

[26] K. Pietruska-Pałuba, Heat kernels on metric spaces and a characterization of constant functions, Manuscripta Math 115 (2004), 389-399.

[27] K. Pietruska-Pałuba, Limiting behaviour of Dirichlet forms for stable processes on metric spaces, Bull. Polish Acad. Sci. Math., to appear.

[28] R. Rammal and G. Toulouse, Random walks on fractal structures and percolation clusters, J. Phys. Lett. 44 (1983), L13-L22.

[29] C. Sabot, Existence et unicité de la diffusion sur un ensemble fractal, C. R. Acad. Sci. Paris Sér. I Math. 321 (1995), 1053-1059.

[30] A. Stós, Symmetric $\alpha$-stable processes on d-sets, Bull. Polish Acad. Sci. Math. 48 (2000), 237-245.

[31] K. T. Sturm, Diffusion processes and heat kernels on metric spaces, Ann. Probab. 26 (1998), 1-55. 
